\title{
Disorders of sex development: Genetic characterization of a patient cohort
}

\author{
MARY GARCÍA-ACERO ${ }^{1}$, OLGA MORENO-NIÑO $^{1}$, FERNANDO SUÁREZ-OBANDO ${ }^{1,2}$, MÓNICA MOLINA $^{1}$, \\ MARÍA CAROLINA MANOTAS ${ }^{1}$, JUAN CARLOS PRIETO ${ }^{1}$, CATALINA FORERO $^{3}$, CAMILA CÉSPEDES $^{3}$, \\ JAIME PÉREZ ${ }^{4}$, NICOLAS FERNANDEZ ${ }^{4}$ and ADRIANA ROJAS ${ }^{1}$ \\ ${ }^{1}$ Instituto de Genética Humana, Facultad de Medicina, Pontificia Universidad Javeriana; ${ }^{2}$ Clinical Genetic Service, \\ Hospital Universitario San Ignacio; ${ }^{3}$ Pediatric Endocrinology; ${ }^{4}$ Division of Urology, Hospital Universitario \\ San Ignacio, Facultad de Medicina, Pontificia Universidad Javeriana, Bogotá 110231, Colombia
}

Received March 28, 2019; Accepted September 9, 2019

DOI: $10.3892 / \mathrm{mmr} .2019 .10819$

\begin{abstract}
Disorders of sex development (DSDs) are congenital conditions in which the external appearance of the individual does not coincide with the chromosomal constitution or the gonadal sex. In other words, there is an ambiguous or intermediate condition between the male and female phenotypes of the anatomical sex. These atypical conditions are manifested in several ways, ranging from genital ambiguity to phenotypes that are so attenuated that they can go unnoticed or appear normal. Currently, there is a lack of understanding of the factors responsible for these outcomes; however, they are likely to be conditioned by genetic, hormonal and environmental factors during prenatal and postnatal development. The present study determined the genetic etiology of DSDs in Colombian patients by conventional cytogenetic analysis, FISH and MLPA (for SF1, DAX1, SOX9, SRY and WNT4). A cohort of 43 patients with clinical phenotypes of sex development disorder was used in the present study. Using this multistep experimental approach, a diagnostic percentage of $25.58 \%$ was obtained: 17 patients $(39.53 \%)$ were classified as having gonadal development disorders, the majority of which were ovotesticular disorders with numerical and/or structural alterations of the sex chromosomes, 9 patients (20.93\%) were classified as having testicular DSD with a 46,XY karyotype, and 3 patients $(6.98 \%)$ as having ovarian DSD with a $46, \mathrm{XX}$ karyotype. The remaining 14 patients $(32.56 \%)$ were classified as 'other' since they could not be grouped into a specific
\end{abstract}

Correspondence to: Dr Adriana Rojas, Instituto de Genética Humana, Facultad de Medicina, Pontificia Universidad Javeriana, Building 32, Cra. 7 No. 40-62, Bogotá 110231, Colombia

E-mail: rojas-adriana@javeriana.edu.co

Abbreviations: MLPA, multiplex ligation-dependent probe amplification; FISH, fluorescence in situ hybridization

Key words: disorders of sex development, gonadal dysgenesis, targeted genetic diagnosis class of gonadal development, corresponding to hypospadias and multiple congenital anomalies. These findings highlight the importance of histological and cytogenetic studies in a gonadal biopsy. In 11/43 cases, the multistep experimental protocol presented in the present study yielded etiological or histological findings that could be used to define the medical management of patients with DSDs. In conclusion, for the etiological diagnosis of DSDs, a broad-spectrum approach that includes endocrinological tests, conventional karyotyping, molecular karyotyping by FISH and, molecular tests is required, in addition to gonadal tissue analyses, to identify genetic alterations.

\section{Introduction}

Disorders of sex development (DSDs) are congenital medical conditions in which there is no correlation between the chromosomal, gonadal, and phenotypic characteristics (1). The term DSDs encompasses a broad clinical spectrum that can be diagnosed at a range of ages, from the neonatal period to late adulthood, the latter of which is often the case with infertility. This condition varies clinically from genital ambiguity to attenuated forms, such as mild hypospadias or unilateral cryptorchidism; this makes it difficult to classify patients with similar or almost identical phenotypes based on different etiologies and molecular processes $(2,3)$. The Lawson Wilkins Pediatric Endocrine Society (LWPES) and the Chicago Consensus in 2006 classified DSDs into three distinct groups: i) DSD 46,XY; ii) DSD 46, and iii) XX DSD, with a certain degree of overlap between the groups $(4,5)$.

Although truly ambiguous genitalia are relatively rare, their prevalence is estimated to be approximately 1 in 4,500-5,500 worldwide (6). However, if we take into account all congenital genital anomalies, including cryptorchidism and hypospadias, then the prevalence ranges from 1:200 to 1:300 (7). In Colombia, the prevalence is 1.7 per 10,000 births (8).

Sex-specific gonadal development starts with formation of the bipotential gonad, which then differentiates into either testicular or ovarian tissue. This process is dependent on activation of either the testis- or ovary-specific pathway, with parallel repression of the opposite pathway. 
For the activation of these sexual differentiation pathways to occur, the transcription factors that regulate the expression of tissue-specific genes and signaling molecules must be expressed (4). Alterations in the molecular processes of these signaling pathways lead to the development of DSDs.

The activation of the male sexual differentiation cascade in mammals depends on the expression of certain genes, such as $S R Y$ (Y sex-determining region), NR5A1, SOX9, and DAXI (in hemicigosis), among others (9); whereas in the activation of the female differentiation cascade, genes including WNT4, $D A X 1$ (in homozygosis), and RSPOI can intervene (10). Given the importance of the correct regulation of these genes, mutations in unidentified enhancer sequences and in the coding regions of these genes, as well as other anomalies, such as total loss of the gene, are important factors that can contribute to the onset of DSDs in humans (11). However, despite advances in their molecular diagnosis, the etiology of DSDs has been established in less than $20 \%$ of cases (12-14), due to both the complexity of the signaling cascades that determine sexual differentiation and the technical limitations of etiological studies.

The etiological diagnosis of DSDs generally requires a broad spectrum of endocrinological tests, radiological images, and genetic tests. Karyotyping is an initial test that allows for the classification of the disorder, according to Lawson Wilkins, $2006(3,15)$. Providing patients with DSDs with a molecular diagnosis allows for the establishment of clinical management techniques, including providing patients with information regarding the risks of neoplasia associated with some types of DSDs (16), as well as offering advice to the patients and their family members regarding recurrence risks.

In Colombia, as well as on a global scale, there are few cytogenetic and molecular studies on patients with DSDs that include stepwise analyses using different genetic techniques. Characterizing the chromosomal and/or molecular alterations and analyzing their contribution to the phenotypes of patients with DSDs could improve our understanding of the causes of these clinical conditions, thus helping in improving diagnosis techniques to allow for specific treatments and medical advice to be given to patients and their families. In this study, we present the cytogenetic and molecular results for 43 Colombian patients with non-syndromic DSDs. The genetic analyses included karyotyping, FISH for $S R Y$, and evaluation of copy number variation in $S F 1, D A X 1, S O X 9$, $S R Y$, and WNT4 genes in the blood and gonadal tissue. Using this multistep experimental approach, a diagnostic percentage of $25.58 \%$ was obtained, which highlights the importance of the histological and cytogenetic study of gonadal biopsies. Our protocol contributed to the diagnosis of 11 out of the 43 patients, for whom the etiology or histological findings allowed for the definition of medical management techniques.

\section{Materials and methods}

Patients. A cohort of 43 individuals with non-syndromic DSDs, aged between 2 days and 49 years, were evaluated by the transdisciplinary board of the Hospital San Ignacio, Bogotá (Colombia). The team consisted of pediatric urologists, pediatric endocrinologists, psychiatrists, clinical geneticists, and cytogeneticists. The cohort consisted of patients with genital ambiguity or discordance between chromosomal, gonadal, and/or phenotypic sex, or genital abnormalities, such as cryptorchidism and hypospadias. Each patient underwent classical cytogenetic analysis, FISH (fluorescent in situ hybridization), and the molecular tests described below.

All evaluations were performed using a step-by-step clinical diagnostic approach. In addition, we provided psychological counseling and advice on the risk of recurrence to both the patients and their families. The genetic analyses included karyotyping, FISH for $S R Y$, and evaluation of the copy number variation in the $S F 1, D A X 1, S O X 9, S R Y$, and WNT4 genes by MLPA in the blood and gonadal tissue. According to the suspected diagnosis, direct sequencing of specific genes was also performed in some individuals.

Declaration of ethics. This research protocol was approved by the Ethics Committee of the Hospital Universitario San Ignacio (FM-CIE-8540) and the School of Medicine of the Pontificia Universidad Javeriana. Patients and their guardians or family members were only included in the study after they had received information regarding the study and signed an informed consent form.

Cytogenetic analysis. Cytogenetic analysis was carried out on all individuals using phytohemagglutinin-stimulated peripheral blood lymphocyte cultures of patients and their parents, according to standard laboratory protocols (17). Gonadal karyotyping was only performed under the treating physician's order. For this, a fragment of the biopsy was processed in culture with RPMI and 20\% SFB for cell growth. Chromosome preparations were treated with $\mathrm{HCl}$ and stained with Wright for G-banding. A total of 50 metaphase cells were analyzed at the 550-band resolution level. Molecular cytogenetics using fluorescence in situ hybridization (FISH) was performed with a probe specific for the SRY gene (SRY Probe, Cytocell Aquarius). The probe mix was obtained according the manufacturer's standard protocol and contained the following: A SRY probe, labelled in red; a control probe for the X centromere (DXZ1), labelled in blue; a control probe for chromosome Y (DYZ1, the heterochromatic block at Yq12) labelled in green; and a centromeric probe for the Y-chromosome (DYZ3 $\alpha$-satellite; Cytocell Aquarius).

Multiplex ligation-dependent probe amplification (MLPA). The deletions and duplications of the SOX9, DAX-1, SF-1, $S R Y$, and WNT4 genes were studied using MLPA SALSA P185-B2 Intersex (version 08; May 07, 2015) (MRC Holland), following the manufacturer's instructions. Information on probe sequences can be freely accessed on the MRC Holland website (www.mlpa.com).

After 35 cycles of PCR amplification, the PCR products were separated using an ABI 3100 genetic analyzer. Row data was analyzed using Coffalyser software (MRC Holland ${ }^{\circledR}$ ). For each sample, the peak areas corresponding to each probe were normalized to the average of the peak areas in the three controls. DNA samples showing a reduction or increase in the MLPA peak area values were reanalyzed using the same MLPA procedure, and only the samples showing consistent results between the two experiment replicates were considered positive for copy number alteration. 
Sequencing. The identification of point mutations in the DNA of the $A R$ and $A M H$ genes was performed using PCR followed by direct sequencing. Sanger sequencing was performed using the BigDye Terminator v1.1 cycle sequencing kit (Thermo Fisher Scientific, Inc.) in a 3500xl genetic analyzer (Thermo Fisher Scientific, Inc.). The direct and inverse sequences were analyzed and compared with each gene's mRNA reference sequence: AR (NM_000044.2) and AMH (NM_000479).

Histological study in gonadal tissue. Based on the recommendations made by the multidisciplinary board, a gonadal biopsy (or gonadectomy) was performed on patients with non-concordant cytogenetic findings, an altered gonadal hormonal study, or whose gonads could not be visualized in diagnostic images. In total, gonadal biopsy samples were obtained from 12 patients.

\section{Results}

Classification of patients with DSD. Cytogenetic, clinical, and molecular techniques were used to classify each patient by syndromic, etiological, and cytogenetic factors (Table I; Fig. 1). Seven patients had a 46,XX karyotype, 30 patients had a 46,XY karyotype, and 6 had a karyotype with sex chromosome aneuploidies that included numerical and/or structural anomalies. The detection of the absence or presence of the $S R Y$ gene in the XY and XX individuals, respectively, did not identify any alterations in relation to the chromosomal complement. Based on additional clinical investigations of the 43 patients, 17 patients $(39.53 \%)$ were classified as having gonadal development disorders, most of which were ovotesticular disorders with numerical and/or structural alterations of the sex chromosomes; 9 patients $(20.93 \%)$ were classified as having testicular DSDs with a 46,XY karyotype; 3 patients $(6.97 \%)$ were classified as having ovarian DSDs with a $46, \mathrm{XX}$ karyotype, and 14 patients $(32.55 \%)$ individuals were classified as 'others' since they could not grouped into a specific class of gonadal development, corresponding to hypospadias and multiple congenital anomalies.

Disorders of gonadal development. The histological findings indicated that 11 individuals with gonadal development alterations had ovotesticular gonads (Fig. 1). Mosaicism was identified in one individual with a male phenotype (case 1) by the presence of $92, \mathrm{XXXX} / 46, \mathrm{XX}$ tetraploidy in the gonadal tissue (Fig. 2A). The presence of an isodicentric Y chromosome was detected in 4 individuals ( 3 males and 1 female: cases 2, 3, 4, and 5). Mosaicism with X chromosome structural and numerical anomalies One was found in an individual with the female phenotype (46,X,del(X) (q21) [71]/45,X[29]. ish Yp11.31 (SRY-) (case 6). Five individuals with ovotestis had XY or XX chromosomal complement without alterations (cases 7, 8, 9, 10, and 11). Two individuals had gonadal strip and testicular regression syndrome with a 46,XY karyotype (cases 12 and 13). Two sister patients with a female phenotype had a 46,XY karyotype with duplication of DAX1 (Fig. 2B), and who had been diagnosed with teratoma at an early age (cases 14 and 15). An adult patient with primary amenorrhea with a 46,XY karyotype had an alteration of $S R Y$, as evidenced by MLPA (case 16). Finally, one individual with a male sex phenotype and a mosaic karyotype 46,X,idic(Y)(q11.2) [38]/45,X[12].ish idic(Y) (SRYx2,DYZ1x0) (case 17) (Fig. 2C).

46,XY testicular DSD. 46,XY testicular DSD was diagnosed in two individuals with partial androgen insensitivity by biochemistry, without molecular confirmation (cases 18 and 19), as well as in a male subject with total androgen insensitivity, with molecular confirmation of a homozygous pathogenic variant not previously reported c.231-239delgcafcagca (p.gln78_gln80del) in the $A R$ gene (case 20). An infertile male phenotype individual was also diagnosed, in whom the persistence of Mullerian derivatives was documented with molecular confirmation of a homozygous pathogenic variant not previously reported c.916delC (p.Leu306Cysfs*29) in the AMHR2 gene (case 21). On the other hand, for 5 of the 9 patients diagnosed with $46, \mathrm{XY}$ testicular DSD, no genetic alterations were found using the proposed approach (cases 22, $23,24,25$, and 26).

46,XX ovarian DSD. 46,XX ovarian DSD was diagnosed in a male patient with atypical congenital adrenal hyperplasia using their biochemical profile, without molecular confirmation (case 28). Two twin brothers with assigned male sex were also diagnosed with 46,XX ovarian DSD, with 46,XX and 46,XY karyotypes, but did not show any molecular alterations using the proposed approach.

Other. We did not find any genetic alterations in 14 patients with hypospadias and multiple congenital anomalies (cases 27-43).

The step-by-step approach with which genetic alterations were found in 11 of the 43 patients $(25.58 \%)$ is shown in Fig. 1.

\section{Discussion}

DSDs affect 1:150 individuals between the neonatal period and adulthood, with various phenotypes and degrees of severity, however all have a significant psychological and clinical impact. It is often difficult to provide patients with DSDs with a molecular diagnosis due to the great clinical heterogeneity of these disorders, as well as our poor understanding of the genetic mechanisms involved in sex development (18). The performances of previously reported different diagnostic approaches range from 13 to $64 \%$ (18-20). In this study, we presented a multistep approach for the diagnosis of DSDs based on blood and gonadal tissue analyses by means of basic and molecular cytogenetics, as well as the detection of copy number variation, which yielded a diagnostic rate of $25.58 \%$. These results highlight the importance of histological and cytogenetic studies in gonadal biopsy, which helped to define the medical management of $11 / 43$ patients based on the resulting etiological or histological findings (Table II).

Eleven patients $(11 / 43,25.58 \%)$ were clinically diagnosed with hypospadias. In most cases, the genetic etiology of this pathology is not established (21). Hypospadias are genital malformations that occur predominantly in individuals with a 46,XY karyotype. The urinary meatus and urethra in individuals with hypospadias is anomalously located in relation to the normal male genital phenotype (22). In patients with this condition, it is necessary to combine all the diagnostic strategies in order to establish their etiology. However, previous 


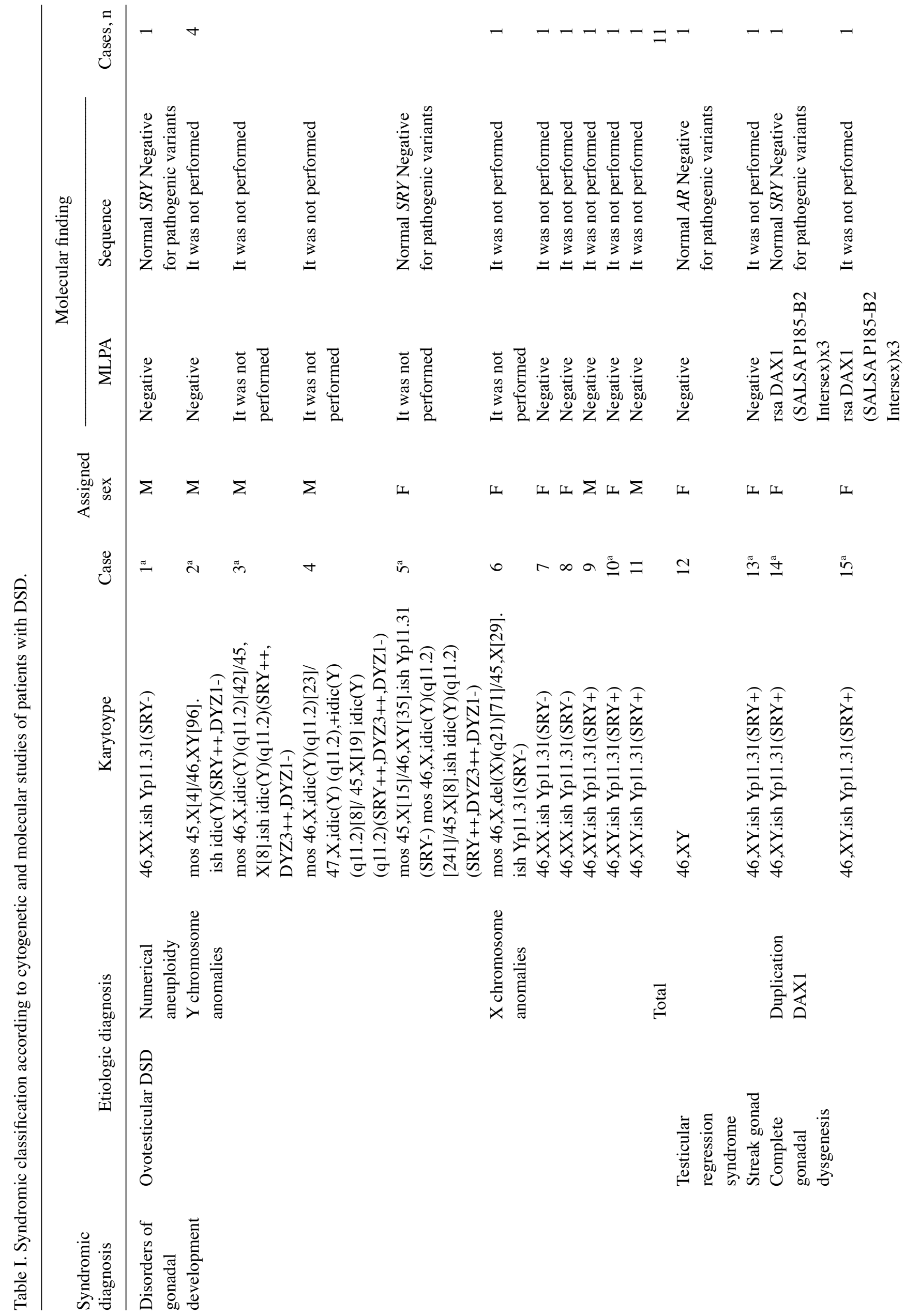




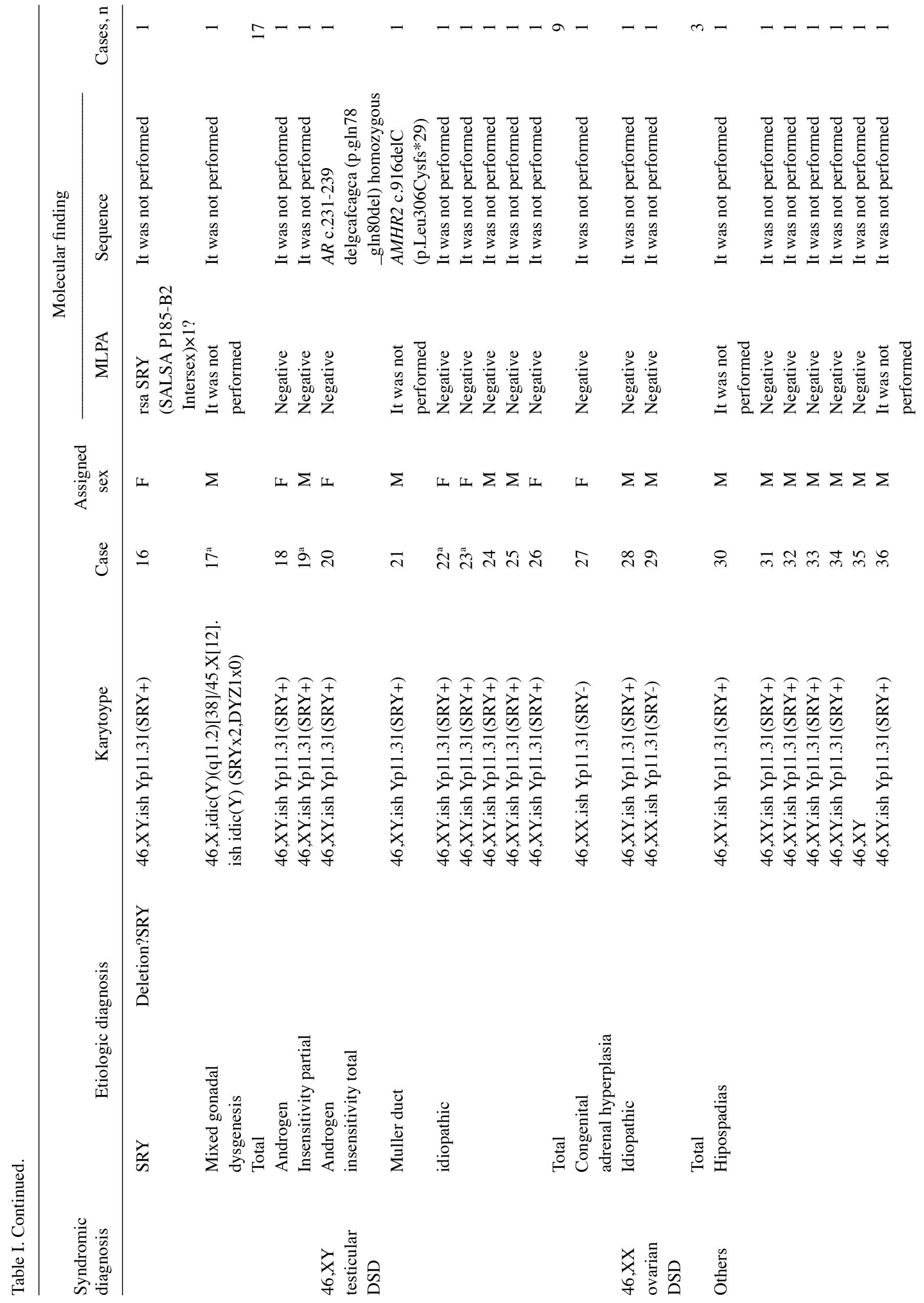




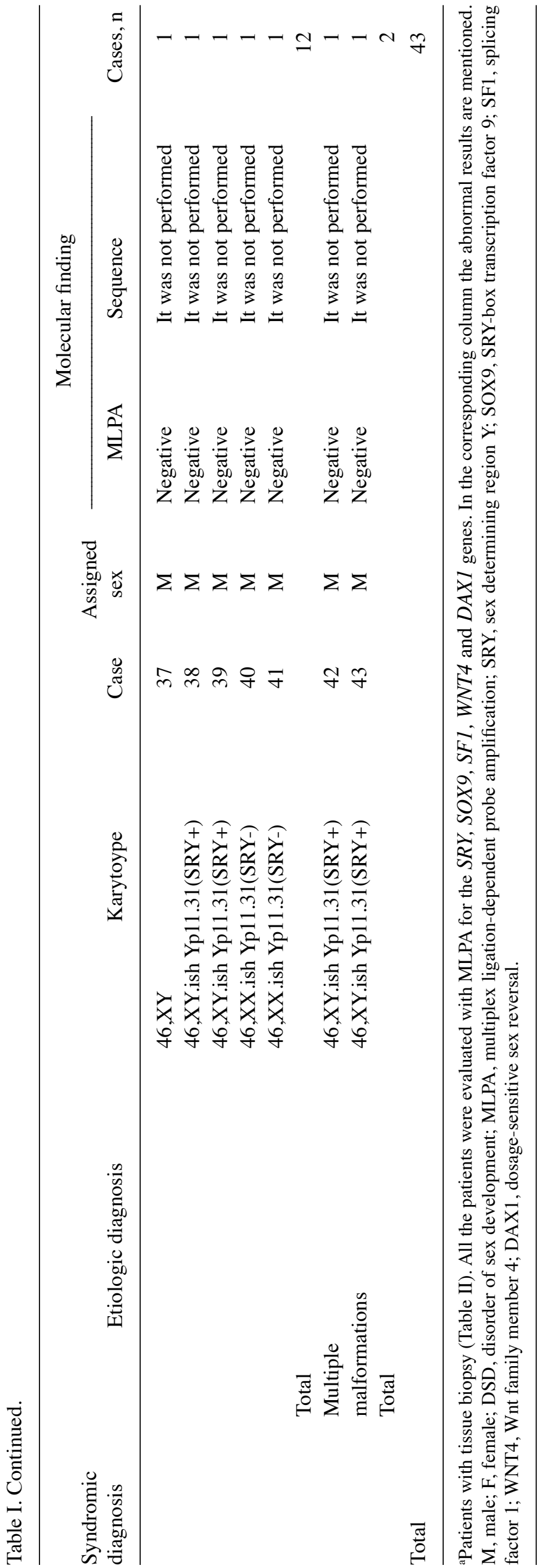




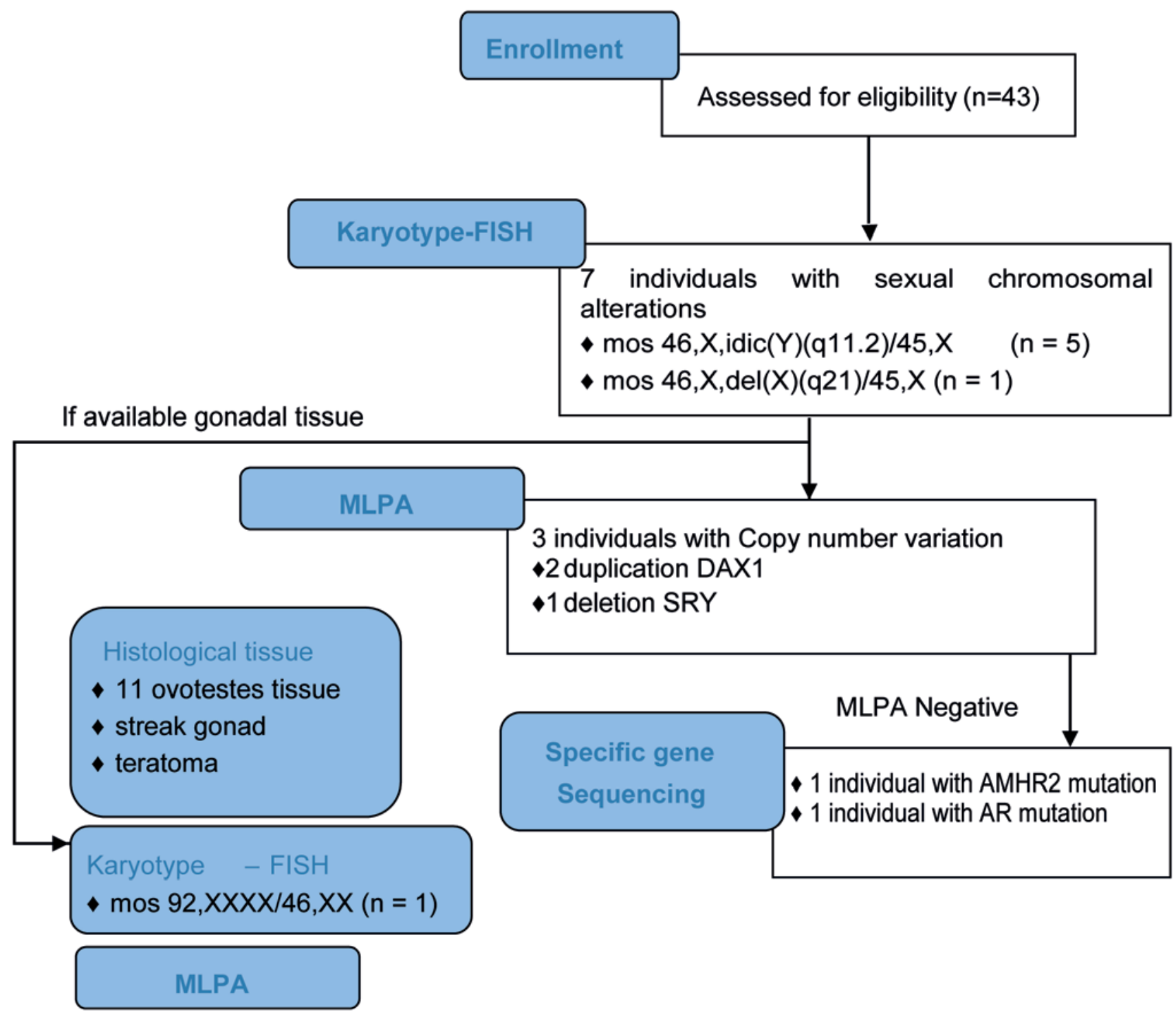

Figure 1. Diagnostic algorithm: Multistep approach of a cohort in a Colombian hospital. The diagram shows the different cytogenetic and molecular tests performed on the patients recruited to the present study. Through the karyotype and FISH assays, 7 patients with chromosomal abnormalities were established, 6 patients with alterations in the number of copies detected by MLPA and 2 patients with point mutations defined by specific gene sequencing. Similarly, cytogenetic and molecular tests performed on gonadal tissue are shown. AMHR2, anti-Mullerian hormone receptor type 2; AR, androgen receptor; DAX1, dosage-sensitive sex reversal; FISH, fluorescence in situ hybridization; MLPA, multiplex ligation-dependent probe amplification; SRY, sex determining region $\mathrm{Y}$.

reports have shown that in over $50 \%$ of cases it was not possible to prove the existence of any genetic or chromosomal cause, such that these patients were classified as having an unknown, or idiopathic, etiology $(22,23)$. Our findings correlate with these results, such that genetic analysis of another series of genes was carried out for this group of patients, in order to determine each patient's phenotype. We chose to investigate genes that are known to play an important role in the development of the urogenital system, such as WTI and SF1 (22). In 2001, Köhler et al (24) reported the presence of mutations in SF1 to be the cause of severe penoscrotal hypospadias and cryptorchidism. Then, in 2004, Wang et al (25) demonstrated that mutations in WT1 were responsible for the development of certain sex development disorders, including penoscrotal hypospadias and micropenis.

The karyotyping performed in this study demonstrated that 7 out of 43 patients $(16.27 \%)$ had an abnormality in their mosaic sex chromosomes. When confirming the FISH results using commercial probes for the $S R Y$ (Yp11.31), DYZ1 (Yq12), DYZ3 (Xp11.1-q11.1), and DXZ1 (Xp11.1-q11.1) regions, we found that the mosaicisms included 45,X, 46,XY, and 47,XYY cell lines and the presence of isodicentric $\mathrm{Y}$ chromosomes. It is worth noting that the phenotypic effect of these mosaics is highly variable, which can be explained by: (1) Difference in the Y chromosome breakpoints, which creates variability in the genetic information that is lost; (2) idic(Y), an unstable chromosome, which is prone to loss during the division of mitotic cells; and (3) different percentages of tissue distribution of mosaicism (26). In addition, a 10-24\% risk of gonadoblastomas has been previously demonstrated in $\operatorname{idic}(\mathrm{Y})$ patients with dysgenetic gonads, which, together with the results described here, justifies the implementation of cytogenetic analyses (both conventional and molecular) as first-line tests, since they are able to detect the frequent genetic causes of DSDs (18). 
Table II. Results of cytogenetic and molecular studies in gonads of patients with developmental sex disorder.

Blood

\begin{tabular}{|c|c|c|c|c|}
\hline & & \multirow[b]{2}{*}{ MLPA } & \\
\hline Case & Karyotype and FISH & & Normal & Abnorma \\
\hline 1 & GD: $\operatorname{mos} 92, \mathrm{XXXX}[8] / 46, \mathrm{XX}[42]$ GI: & It was not performed & Table I & \\
\hline 2 & $\operatorname{mos} 45, \mathrm{X}[97] / 46, \mathrm{XY}[3]$.ish $\operatorname{idic}(\mathrm{Y})(\mathrm{SRY}++, \mathrm{DYZ1}-)$ & It was not performed & & Table I \\
\hline 10 & 46,XY.ish Yp11.31(SRY+) & Negative & Table I & \\
\hline 22 & 46,XY.ish Yp11.31(SRY+) & It was not performed & Table I & \\
\hline 14 & Not performed & $\begin{array}{l}\text { Dup Dax1 rsa DAX1 } \\
\text { (SALSA P185-B2 Intersex)x } 3\end{array}$ & Table I & \\
\hline 15 & Not performed & $\begin{array}{l}\text { Dup Dax1 rsa DAX1 } \\
\text { (SALSA P185-B2 Intersex)x3 }\end{array}$ & Table I & \\
\hline 13 & 46,XY.ish Yp11.31(SRY+) & Negative & Table I & \\
\hline 23 & GI: 46,XY[50]SRY (+) & It was not performed & Table I & \\
\hline 19 & GD: 46,XY[36]/92,XXYY[14] & & & \\
\hline & GI: $46, X Y[40] 92, X X Y Y[10]$ & It was not performed & Table I & \\
\hline 3 & $\begin{array}{l}\operatorname{mos} 46, \mathrm{X}, \operatorname{idic}(\mathrm{Y})(\mathrm{q} 11.2)[42] / 45, \mathrm{X}[8] \text {.ish idic } \\
(\mathrm{Y})(\mathrm{q} 11.2)(\mathrm{SRY}++, \mathrm{DYZ}++, \mathrm{DYZ1}-)\end{array}$ & It was not performed & & Table I \\
\hline 5 & $\begin{array}{l}\operatorname{mos} 45, \mathrm{X}[15] / 46, \mathrm{XY}[35] . \text { ish Yp11.31(SRY-) } \\
\operatorname{mos} 46, \mathrm{X}, \operatorname{idic}(\mathrm{Y})(\mathrm{q} 11.2)[241] / 45, \mathrm{X}[8] . \text { ish idic } \\
(\mathrm{Y})(\mathrm{q} 11.2)(\mathrm{SRY}++, \mathrm{DYZ3++,DYZ1-)}\end{array}$ & It was not performed & & Table I \\
\hline 17 & $\begin{array}{l}\operatorname{mos} 45, \mathrm{X}[15] / 46, \mathrm{XY}[35] . i s h \mathrm{Yp} 11.31(\mathrm{SRY}-) \\
\operatorname{mos} 46, \mathrm{X}, \mathrm{idic}(\mathrm{Y})(\mathrm{q} 11.2)[241] / 45, \mathrm{X}[8] . \text { ish idic } \\
(\mathrm{Y})(\mathrm{q} 11.2)(\mathrm{SRY}++, \mathrm{DYZ3++} \text {,DYZ1-) }\end{array}$ & It was not performed & & Table I \\
\hline
\end{tabular}

M, male; F, female; MLPA, multiplex ligation-dependent probe amplification; FISH, fluorescence in situ hybridization.

A

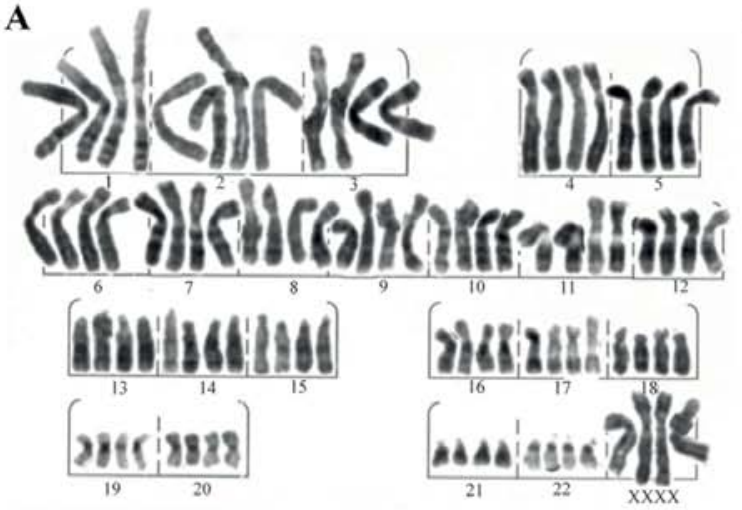

B

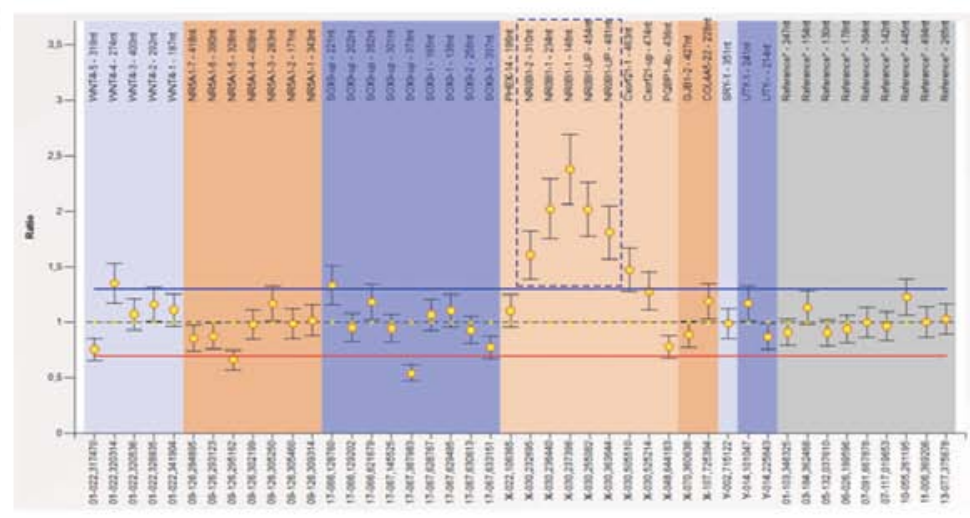

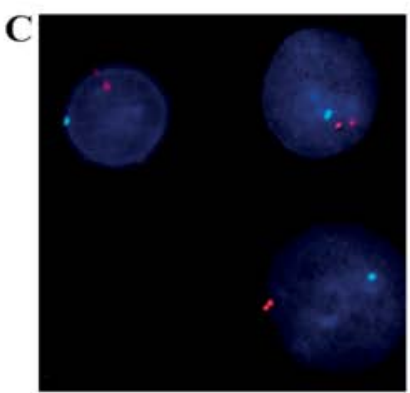

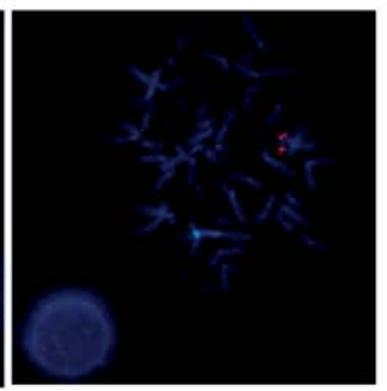

Figure 2. Graphical representation of results obtained through the proposed approach. (A) High resolution G-banding karyotype, 96,XXXX. Magnification, $\mathrm{x} 100$. (B) Result of the multiplex ligation-dependent probe amplification test showing duplication of the DAX1, rsa DAX1(SALSA P185-B2 Intersex) x3 gene. (C) Fluorescence in situ hybridization for $S R Y$ in interphase nuclei (left) and metaphase chromosomes (right) of a patient exhibiting double signal for $S R Y$ (red). This probe contained a blue probe that recognizes the DXZ1 region of the centromere of the X, mos 45,X[4]/46,XY[96] idic?(Y)(q11.2)(SRY++,DYZ1-). Magnification, x100. DAX1, dosage-sensitive sex reversal; SRY, sex determining region Y. 
MLPA analysis detected copy number variations in 3 patients: 2 patients, who were sisters, showed duplication of $D A X 1$ and a 46,XY karyotype, suggesting that the causal alteration for DSDs in these patients was inherited from a parent with gonadal mosaicism; although this is a very rare characteristic, it has been previously described in a patient with DSDs, with copy number variation in another chromosomal region $(27,28)$. In the third patient, the deletion of the $S R Y$ gene region with a $46, X Y$ karyotype was demonstrated; in this case, the presence of $S R Y$, as detected by FISH, suggests the probable alteration of the gene by mutation in the annealing region, which has an effect on the function of the gene, and is likely to be the cause of the DSDs phenotype (29).

Sequencing analysis of specific genes resulted in the identification of a pathogenic variant in the $A R$ gene, which caused total androgen insensitivity, and a homozygous pathogenic variant in the AMHR2 gene in an infertile male-phenotype individual with persistent Mullerian derivatives. In both cases, the clinical, endocrine, histopathological, and cytogenetic data were consistent with the results of the molecular study. This suggests that the de novo mutations detected in the $A R$ and $A M H R 2$ genes are etiopathogenic factors in several DSDs, including partial androgen insensitivity syndrome (MIM 312300) and persistent Müllerian duct syndrome (MIM 261550).

In 6 of the 9 patients with 46,XY testicular DSD and 2 of the 3 patients with $46, \mathrm{XX}$ ovarian DSD, no genetic alteration was found using the proposed diagnostic approach. This suggests that other molecular mechanisms were responsible for the DSDs in these patients. Unexplained cases of $S R Y$-negative XX male reversal in humans may arise from loss-of-function mutations in the pro-ovarian pathway (such as in canonical WNT signaling components) or gain-of-function mutations in genes whose products mimic SRY activity, of which members of the SOX gene family are good candidates $(30,31)$.

Additionally, a histological study of the gonadal tissues from 13 patients was performed. Eleven of the 13 patients were diagnosed with ovotesticular DSD, which is associated with different anomalies in the mosaic sex chromosomes, including mosaicism in gonadal tissue (Table II). One out of the 13 patients was diagnosed with streak gonad. In general, this type of gonadal abnormality is observed in patients with pure and syndromic gonadal dysgenesis, which is associated with the development of gonadoblastoma (32-34).

It is highlighted, despite the group of patients that had undergone gonadectomy and subsequent histopathologic examination the clinical decision was not based on the our propose algorithm, in the near future this diagnostic algorithm will help to focus genetics investigation and in some case could improve clinical management. For example, in case of detection of an abnormal Y chromosome with classic cytogenetic analysis complemented with FISH techniques or detection of gene dosage imbalances using MLPA in patients with 46,XY gonadal dysgenesis with implications in sex determination and development of gonadal tumors, the identification of the molecular cause can be very helpful in decision-making such as prophylactic gonadectomy (35).

Although an etiological diagnosis may not affect the clinical management of many cases of DSDs, diagnosis allows patients with rare chronic disorders to anticipate the health-related and psychological effects of their condition to optimize their quality of life (36). Additionally, the diagnosis of genetic etiologies plays an important role in the genetic counseling of parents, children, and other family members (37). The clinical management of all patients with DSDs requires a multidisciplinary team, since the issues associated with DSDs are multidimensional and thus require the cooperation of a number of disciplines in order to provide an effective diagnosis, treatment, and support. An ideal management team includes pediatric subspecialists in endocrinology, surgery and/or urology, psychology or psychiatry, gynecology, genetics, neonatology, and, if available, social workers and nurses, among others. This multidisciplinary team plays a critical role in the provision of care and has as its aim the physical and psychological well-being of individuals with DSDs and their families $(37,38)$.

Karyotyping serves as an initial test, the results of which lead to additional tests, as it allows for zeroing in on a specific diagnosis from possible diagnoses. In this context, the study of sequence variants and copy number variations is necessary, wherein a sequencing approach with an algorithm for the detection of copy number variants would be a good option. However, it is worth bearing in mind that DNA modifications are not the only possible explanation for DSDs, and thus epigenetic modifications should be taken into account in the approach. Finally, to determine tissue-specific expression, this type of study must be performed using gonadal tissue.

Our step-by-step approach in individuals with DSDs combines the cost-effectiveness of molecular cytogenetics with the study of copy number variation to provide an effective algorithm for the genetic diagnosis of patients with DSDs to improve our understanding of the genetic etiology in a heterogeneous cohort of patients. Our findings demonstrate the utility of a gonadal biopsy in male- and female-phenotype individuals with DSDs.

\section{Acknowledgements}

Not applicable.

\section{Funding}

The present study was supported by COLCIENCIAS (grant no. 711/2015; project no. 120371150037). MGA also received a Young Researcher grant from COLCIENCIAS (grant no. $761 / 2016)$.

\section{Availability of data and materials}

The datasets used and/or analyzed during the present study are available from the corresponding author on reasonable request.

\section{Authors' contributions}

MGA, MM, OMN and AR designed and performed the experiments and analyzed the data. MCM, FS, JCP, CF, CC, JP and NF provided technical and conceptual advice and analyzed the results. AR and OMN supervised the research and analyzed the data. MGA, MCM and AR wrote the main parts of the manuscript. 


\section{Ethics approval and consent to participate}

This research protocol was approved by the Ethics Committee of the Hospital Universitario San Ignacio (approval no. FM-CIE-8540) and the School of Medicine of the Pontificia Universidad Javeriana.

\section{Patient consent for publication}

The data and results presented in the present study were anonymized and patients provided consent for publication.

\section{Competing interests}

The authors declare that they have no competing interests.

\section{References}

1. Woodward MN and Patwardhan N: Disorders of sex development. Surg 28: 396-401, 2010.

2. Dreger AD, Chase C, Sousa A, Gruppuso PA and Frader J: Changing the nomenclature/taxonomy for intersex: A scientific and clinical rationale. J Pediatr Endocrinol Metab 18: 729-33, 2005.

3. Hughes IA: Disorders of Sexual Differentiation. Horm Res Paediatr 67: 91-95, 2007.

4. Lee PA, Nordenström A, Houk CP, Ahmed SF, Auchus R, Baratz A, Baratz Dalke K, Liao LM, Lin-Su K, Looijenga LH III, et al: Global disorders of sex development update since 2006: Perceptions, approach and care. Horm Res Paediatr 85: 158-180, 2016.

5. Hughes IA, Houk CP, Ahmed SF and Lee PA; LWPES Consensus Group; ESPE Consensus Group: Consensus statement on management of intersex disorders. Arch Dis Child 91: 554-563, 2006.

6. Sax L: How common is lntersex? A response to Anne Fausto-Sterling. J Sex Res 39: 174-178, 2002.

7. Nordenvall AS, Frisén L, Nordenström A, Lichtenstein P and Nordenskjöld A: Population based nationwide study of hypospadias in sweden, 1973 to 2009: Incidence and risk factors. J Urol 191: 783-789, 2014.

8. Zarante I, Franco L, López C and Fernández N: Frecuencia de malformaciones congénitas: Evaluación y pronóstico de 52.744 nacimientos en tres ciudades colombianas. Biomédica 30: 65-71, 2010.

9. Biason-Lauber A: Control of sex development. Best Pract Res Clin Endocrinol Metab 24: 163-186, 2010.

10. Houmard B, Small C, Yang L, Naluai-Cecchini T, Cheng E, Hassold T and Griswold M: Global gene expression in the human fetal testis and Ovary1. Biol Reprod 81: 438-443, 2009.

11. Eggers S, Sadedin S, van den Bergen JA, Robevska G, Ohnesorg T, Hewitt J,Lambeth L, Bouty A, Knarston IM, Tan TY, et al: Disorders of sex development: Insights from targeted gene sequencing of a large international patient cohort. Genome Biol 17: 243, 2016.

12. McClelland K, Bowles J and Koopman P: Male sex determination: Insights into molecular mechanisms. Asian J Androl 14: 164-171, 2012 .

13. Arboleda VA, Sandberg DE and Vilain E: DSDs: Genetics, underlying pathologies and psychosexual differentiation. Nat Rev Endocrinol 10: 603-615, 2014.

14. Baxter RM and Vilain E: Translational genetics for diagnosis of human disorders of sex development. Annu Rev Genomics Hum Genet 14: 371-392, 2013.

15. Rajfer J and Walsh PC: The incidence of intersexuality in patients with hypospadias and cryptorchidism. J Urol 116: 769-770, 1976

16. van der Zwan YG, Biermann K, Wolffenbuttel KP, Cools M and Looijenga LH: Gonadal maldevelopment as risk factor for germ cell cancer: Towards a clinical decision model. Eur Urol 67: 692-701, 2015.

17. Moorhead PS, Nowell PC, Mellman WJ, Battips DM and Hungerford DA: Chromosome preparations of leukocytes cultured from human peripheral blood. Exp Cell Res 20: 613-616, 1960.

18. Laino L, Majore S, Preziosi N, Grammatico B, De Bernardo C, Scommegna S, Rapone AM, Marrocco G, Bottillo I and Grammatico P: Disorders of sex development: A genetic study of patients in a multidisciplinary clinic. Endocr Connect 3: 180-192, 2014.
19. Arboleda VA, Lee H, Sánchez FJ, Délot EC, Sandberg DE, Grody WW, Nelson SF and Vilain E: Targeted massively parallel sequencing provides comprehensive genetic diagnosis for patients with disorders of sex development. Clin Genet 83: 35-43, 2013.

20. Dong Y, Yi Y, Yao H, Hu H, Liu J, Gao C, Zhang M, Zhou L, Asan, Yi X and Liang Z: Targeted next-generation sequencing identification of mutations in patients with disorders of sex development. BMC Med Genet 17: 23, 2016.

21. Marrocco G, Grammatico P, Vallasciani S, Gulia C, Zangari A, MarroccoF, BateniZH,Porrello A and Piergentili R:Environmental, parental and gestational factors that influence the occurrence of hypospadias in male patients. J Pediatr Urol 11: 12-19, 2015.

22. Audí L and Fernández-Cancio MF: Etiopatogenia del hipospadias. Rev Esp Endocrinol Pediatr 5 (Suppl): S7, 2014.

23. Si YM, Dong Y, Wang W, Qi KY and Wang X: Hypospadias in a male infant with an unusual mosaic $45, \mathrm{X} / 46, \mathrm{X}$,psu idic(Y) (p11.32)/46,XY and haploinsufficiency of SHOX: A case report. Mol Med Rep 16: 201-207, 2017.

24. Köhler B, Schumacher V, L'Allemand D, Royer-Pokora B and Grüters A: Germline Wilms tumor suppressor gene (WT1) mutation leading to isolated genital malformation without Wilms tumor or nephropathy. J Pediatr 138: 421-424, 2001.

25. Wang Y, Li Q, Xu J, Liu Q, Wang W, Lin Y, Ma F, Chen T, Li S and Shen Y: Mutation analysis of five candidate genes in Chinese patients with hypospadias. Eur J Hum Genet 12: 706-712, 2004.

26. Beaulieu Bergeron M, Brochu P, Lemyre E and Lemieux N: Correlation of intercentromeric distance, mosaicism, and sexual phenotype: Molecular localization of breakpoints in isodicentric Y chromosomes. Am J Med Genet A 155A: 2705-2712, 2011.

27. Haines B, Hughes J, Corbett M, Shaw M, Innes J, Patel L, Gecz J, Clayton-Smith J and Thomas P: Interchromosomal insertional translocation at Xq26.3 Alters SOX3 expression in an individual with XX male sex reversal. J Clin Endocrinol Metab 100: E815-E820, 2015.

28. García-Acero M, Molina M, Moreno O, Ramirez A, Forero C, Céspedes C, Prieto JC, Pérez J, Suárez-Obando F and Rojas A: Gene dosage of DAX-1, determining in sexual differentiation: Duplication of DAX-1 in two sisters with gonadal dysgenesis. Mol Biol Rep 46: 2971-2978, 2019.

29. McElreavy K, Vilain E, Abbas N, Costa JM, Souleyreau N, Kucheria K, Boucekkine C, Thibaud E, Brauner R, Flamant F, et al: XY sex reversal associated with a deletion 5' to the SRY 'HMG box' in the testis-determining region. Proc Natl Acad Sci USA 89: 11016-11020, 1992.

30. Sarkar A and Hochedlinger K: The Sox family of transcription factors: Versatile regulators of stem and progenitor cell fate. Cell Stem Cell 12: 15-30, 2013.

31. XIA XY, ZHANG C, LI TF, Wu QY, Li N, Li WW, Cui YX, Li XJ and Shi YC: A duplication upstream of SOX9 was not positively correlated with the SRY-negative 46,XX testicular disorder of sex development: A case report and literature review. Mol Med Rep 12: 5659-5664, 2015.

32. Hashimoto K, Horibe YU, Ezaki J, Kanno T, Takahashi N, Akizawa Y, Matsui H, Yamamoto $T$ and Shibata N: Laparoscopically removed streak gonad revealed gonadoblastoma in frasier syndrome. Anticancer Res 37: 3975-3979, 2017.

33. Mandelberger A, Mathews S, Andikyan V and Chuang L: Laparoscopic removal of streak gonads in turner syndrome. J Minim Invasive Gynecol 23: 1025, 2016.

34. Lepais L, Morel Y, Mouriquand P, Gorduza D, Plotton I, Collardeau-Frachon S and Dijoud F: A novel morphological approach to gonads in disorders of sex development. Mod Pathol 29: 1399-1414, 2016.

35. Achermann JC, Domenice S, Bachega TA, Nishi MY and Mendonca BB: Disorders of sex development: Effect of molecular diagnostics. Nat Rev Endocrinol 11: 478-488, 2015.

36. Délot EC, Papp JC, Délot EC; DSD-TRN Genetics Workgroup, Sandberg DE and Vilain E: Genetics of Disorders of Sex Development: The DSD-TRN experience. Endocrinol Metab Clin North Am 46: 519-537, 2017.

37. Anthony E, Baratz AB, Boney C, et al (eds): Clinical Guidelines for the Management of Disorders of Sex Development in Childhood. 1st edition. Intersex Society of North America, Rohnert Park, CA, 2006.

38. Gomez-Lobo V: Multidisciplinary care for individuals with disorders of sex development. Curr Opin Obstet Gynecol 26: 366-371, 2014.

This work is licensed under a Creative Commons Attribution-NonCommercial-NoDerivatives 4.0 International (CC BY-NC-ND 4.0) License. 